\title{
Signaling of the Strongest Stimulus in the Owl Optic Tectum
}

\author{
Shreesh P. Mysore, Ali Asadollahi, and Eric I. Knudsen \\ Department of Neurobiology, Stanford University, Stanford, California 94305
}

Essential to the selection of the next target for gaze or attention is the ability to compare the strengths of multiple competing stimuli (bottom-up information) and to signal the strongest one. Although the optic tectum (OT) has been causally implicated in stimulus selection, how it computes the strongest stimulus is unknown. Here, we demonstrate that OT neurons in the barn owl systematically encode the relative strengths of simultaneously occurring stimuli independently of sensory modality. Moreover, special "switch-like" responses of a subset of neurons abruptly increase when the stimulus inside their receptive field becomes the strongest one. Such responses are not predicted by responses to single stimuli and, indeed, are eliminated in the absence of competitive interactions. We demonstrate that this sensory transformation substantially boosts the representation of the strongest stimulus by creating a binary discrimination signal, thereby setting the stage for potential winner-take-all target selection for gaze and attention.

\section{Introduction}

A causal role for the optic tectum (OT, also called the superior colliculus in mammals) in the control of spatial attention has been firmly established. Focal manipulation of neuronal excitability in the OT systematically alters the competitive advantage for attention of a stimulus represented in the manipulated portion of the OT (Carello and Krauzlis, 2004; McPeek and Keller, 2004; Müller et al., 2005; Cavanaugh et al., 2006; Lovejoy and Krauzlis, 2010). These studies demonstrate the importance of competition in the OT for target selection. However, how the OT represents competing stimuli as a function of their relative strengths remains unknown.

Computational models of attention have suggested that the mechanisms underlying stimulus selection involve the creation of a space map of the relative strengths of competing stimuli (Wolfe, 1994; Itti and Koch, 2001), followed by a winner-take-all process to select the next target for gaze and attention. Such a map has been found to exist in the lateral intraparietal cortex (LIP) and the frontal eye fields in monkeys (Schall and Thompson, 1999; Bisley and Goldberg, 2003). Indirect evidence from several studies has led to the hypothesis that a similar map may also exist in the intermediate and deep layers of the OT (OTi-d) (Fecteau and Munoz, 2006). Neurons in the OTi-d form a topographic map of multisensory space as demonstrated in owls (Knudsen, 1982), cats (Middlebrooks and Knudsen, 1984), and primates (Wurtz and Goldberg, 1971; Wallace et al., 1996). These neurons are highly sensitive to intrinsically salient properties of stimuli in their receptive fields, such as speed of motion or contrast, as shown in owls (Jassik-Gerschenfeld and Guichard, 1972; Hughes and Pearlman, 1974; Mysore et al., 2010) and primates (Mar-

Received Sept. 1, 2010; revised Dec. 16, 2010; accepted Feb. 4, 2011.

This work was supported by a Stanford University School of Medicine Dean's Postdoctoral Fellowship (S.P.M.) and National Institutes of Health Grant 9 R01 EY019179-30 (E.I.K.). We thank Alex Goddard, Cathy Dunn, and Devarajan Sridharan for critically reading this manuscript.

Correspondence should be addressed to Shreesh P. Mysore, Department of Neurobiology, Stanford University, 299 West Campus Drive, Stanford, CA 94305. E-mail: shreesh@stanford.edu.

DOI:10.1523/JNEUROSCI.4592-10.2011

Copyright $\odot 2011$ the authors $\quad 0270-6474 / 11 / 315186-11 \$ 15.00 / 0$ rocco and Li, 1977; Wurtz and Albano, 1980) typically responding with increasing firing rates to larger values. Conversely, they are rarely selective for features such as contour orientation or direction of motion (Li et al., 1996; Horwitz and Newsome, 1999). Moreover, sensory responses in the OTi-d are competitive: responses to stimuli inside the classical receptive fields of OTi-d neurons are inhibited by remote stimuli of the same or different sensory modality, as demonstrated in owls (Mysore et al., 2010), pigeons (Frost et al., 1981), cats (Rizzolatti et al., 1974; Meredith and Stein, 1996), and primates (Basso and Wurtz, 1997; Trappenberg et al., 2001). Despite these indirect lines of evidence and the established role of the OT in competitive stimulus selection, it is unknown whether the OT itself contains a map of relative stimulus strength and, if so, what its properties are.

In this study, we directly address these issues by measuring the representation of the relative strengths of competing stimuli in the barn owl OTi-d. We show that relative neuronal firing rates explicitly encode relative stimulus strength. In addition, "switchlike" responses in a subpopulation of neurons lead to a binary discrimination signal of the strongest stimulus. These bottom-up competitive interactions operate automatically on sensory information (in tranquilized as well as in nontranquilized animals) and set the stage for stimulus selection for attention and gaze.

\section{Materials and Methods}

Neurophysiology. Materials and Methods followed protocols that have been described previously (Mysore et al., 2010). Briefly, tungsten microelectrodes $(250 \mu \mathrm{m}, 1-5 \mathrm{M} \Omega$ at $1 \mathrm{kHz}$; FHC) were used to record single and multi-units extracellularly in nine barn owls (both male and female) that typically were tranquilized with a mixture of nitrous oxide and oxygen. Multi-unit spike waveforms were sorted offline into putative single units (Fee et al., 1996; Mitra and Bokil, 2008). All recordings were made in layers 11-13 of the OT, referred to as the intermediate to deep layers of the OT, or OTi-d.

Stimuli and experimental protocol. Visual stimuli were created using customized MATLAB software (courtesy of J. Bergan, Harvard University, Cambridge, MA) and presented on a tangent screen (full contrast, dark dots on gray background). The expanding (looming) visual stimuli used here and OTi-d responses to looming dots have been described 
previously (Mysore et al., 2010). Briefly, looming stimuli were dots that expanded linearly in size over time, starting from a size of $0.6^{\circ}$ in radius. Responses to looming dots were compared with responses to stationary dots of the same final sizes (and luminance), and it was shown that the speed of a looming stimulus, but not the final size, is the dominant predictor of the responses to this stimulus. Auditory stimuli, delivered dichotically through matched earphones, were presented as though from different locations by filtering sounds with head-related transfer functions (Witten et al., 2010). The average binaural levels (ABLs) (referred to also as sound levels) of auditory stimuli are indicated in all figures relative to the minimum threshold observed across all units.

The experimental protocol used in this study involved the simultaneous presentation of two stimuli: one stimulus, $S_{\text {in }}$, was presented inside the receptive field of the unit and its strength was held constant, whereas the second stimulus, $S_{\text {out }}$, was presented outside the receptive field and its strength was systematically varied. The resulting responses from this protocol were collectively referred to as a competitor strength-response profile (CRP).

The spatial receptive field for each unit was defined as the set of locations at which a single stimulus evoked responses above baseline. The $S_{\text {in }}$ stimulus was presented at the center of the receptive field, and the $S_{\text {out }}$ stimulus was presented outside, $30^{\circ}$ away from the center $\left(30.2 \pm 0.5^{\circ}\right.$, $n=169 ; \mathrm{S}_{\text {out }}$ located between $25^{\circ}$ and $40^{\circ}$ away in azimuth and between $0^{\circ}$ and $10^{\circ}$ away in elevation). By definition of the receptive field, the $S_{\text {out }}$ stimulus alone did not evoke any responses from the unit being recorded (Mysore et al., 2010). For units with frontally located receptive fields (azimuth between $5^{\circ}$ ipsilateral and $20^{\circ}$ contralateral), $\mathrm{S}_{\text {out }}$ was located lateral to the receptive field, and for units with peripherally located receptive fields (azimuth more contralateral than $20^{\circ}$ ), $\mathrm{S}_{\text {out }}$ was located medial to the receptive field (supplemental Fig. S3A, available at www. jneurosci.org as supplemental material). The most medial $\mathrm{S}_{\text {out }}$ location tested was $6^{\circ}$ ipsilateral. Because the barn owl OT represents locations up to $15^{\circ}$ into ipsilateral space (Knudsen, 1982), $\mathrm{S}_{\text {out }}$ locations in our experiments were always represented in the same hemisphere as the $S_{\text {in }}$ locations. The receptive fields of the recorded units ranged in azimuth from $5^{\circ}$ ipsilateral to $43^{\circ}$ contralateral and in elevation from -43 to $+40^{\circ}$. The average center of the receptive field (mean $\pm \mathrm{SD}$ ) was contralateral $10.3 \pm 11^{\circ}$ in azimuth and $-1.3 \pm 22^{\circ}$ in elevation.

The loom speed ("strength") of the $S_{\text {in }}$ stimulus was chosen so that it elicited $\sim 50 \%$ of the maximal response for each unit ( $55 \pm 5 \%$; median speed across 169 units, $7.6 \%$ with $95 \%$ confidence interval of $[6,10 \% \mathrm{~s}]$ ). An $S_{\text {in }}$ loom speed that elicited $\sim 50 \%$ of the maximal response was strong enough to drive strong unit responses, while allowing for the selection of a $6 \%$ s higher, nonsaturating $\mathrm{S}_{\text {in }}$ speed for the measurement of a second CRP (see Fig. 5C-E).

The strength of the looming $\mathrm{S}_{\text {out }}$ stimulus was varied across the range of $0-22 \%$. This was determined in a previous study to be the range of loom speeds over which OTi-d neurons are most sensitive (Mysore et al., 2010). The strength of the auditory $S_{\text {out }}$ stimulus was varied across the range of $0-50 \mathrm{~dB}$ relative to unit threshold.

Data analysis. All analyses were performed with custom MATLAB code. Response firing rates were computed by counting spikes over a $100-250 \mathrm{~ms}$ window with respect to stimulus onset and converting the resulting count into spikes per second. Parametric or nonparametric statistical tests were applied based on whether the distributions being compared were Gaussian or not (Lilliefors test of normality), and corrections for multiple comparisons were applied when appropriate. Data shown as $a \pm b$ refer to mean $\pm \mathrm{SEM}$, unless otherwise indicated.

Correlations between responses to paired stimuli and the strength of the $S_{\text {out }}$ stimulus were tested using Spearman's rank correlation coefficient (corr command in MATLAB with the Spearman option). Units that did not show a significant correlation $(p>0.05$, correlation test) were considered to exhibit fixed response suppression if the suppression was significant for most values of the $\mathrm{S}_{\text {out }}$ stimulus $(p<0.05$, rank-sum test against response to $S_{\text {in }}$ alone followed by the Holm-Bonferroni correction). The remaining units (for which the responses were neither correlated with $S_{\text {out }}$ strength nor suppressed by a fixed amount) were considered as showing no effect of $\mathrm{S}_{\text {out }}$. Best sigmoidal fits to CRPs were obtained by fitting the sigmoidal equation $r(x)=c+s /\left(1+e^{-m(-d)}\right)$ to the data, using a nonlinear least squares estimation procedure (nlinfit command in MATLAB). Here, $r(x)$ is the baseline-subtracted average response at a loom speed of $x \% \mathrm{~s}, c$ is the minimum response, $s$ is the maximum response, $m$ is the slope parameter, and $d$ is the speed at which the slope of the function was maximum (also the speed at which the response was halfway between maximum and minimum). Best linear fits were also obtained using an identical procedure but by fitting the linear equation $r(x)=m x+c$ to the data, where $m$ is the slope and $c$ is the $y$-axis intercept. The best fit of the two models was selected using Akaike's information criterion (AIC), which quantifies the goodness-of-fit of a model while penalizing for the number of model parameters. It is defined as AIC $=2 * k+n \ln (\mathrm{RSS} / n)$, where $k$ is the number of parameters in the model, $n$ is the number of data points, and RSS is the sum of squared residuals. A lower AIC value indicates a better fit of the model to the data. In the text, we also report the $r^{2}$ values of the resulting best-fit function.

The transition range of a CRP was defined as the range of $\mathrm{S}_{\text {out }}$ loom speeds over which responses dropped from 90 to $10 \%$ of the total range of responses. The minimum and maximum response rates were estimated over the range of $0-22 \%$ for looming visual stimuli and $0-50 \mathrm{~dB}$ relative to unit threshold, for auditory burst stimuli. These ranges were previously determined to be the ranges over which most OTi-d neurons are sensitive to the value of the stimulus feature. The upper and lower $95 \%$ prediction bounds for the best-fit function (Fig. 1 B2,C2) were determined using the predint function in MATLAB. Response pooling across units was achieved by first normalizing the responses to the maximum response for each unit and then binning the combined responses across all units along the $x$-axis variable. The binning was performed in MATLAB using the hist command.

The switch value for switch-like CRPs was defined as the strength of the $S_{\text {out }}$ stimulus at which responses to the paired stimuli changed abruptly from high to low. It was estimated as the $S_{\text {out }}$ strength at which the slope of the best-fit sigmoid was maximal (the parameter $d$ ) and was numerically equal to the midpoint of the transition range of the CRP. In experiments in which CRPs were obtained with different $S_{\text {in }}$ strengths (see Fig. $3 D, E$ ), the best-fit sigmoid to one CRP and its switch value were determined. The data from the second CRP were then best fit to two sigmoidal equations. In case I ("best unconstrained fit"), all four parameters of the sigmoidal equation were treated as free parameters and the best fit was obtained, which yielded the switch value of the second CRP. In case II ("best constrained fit"), the parameter $d$ of the sigmoidal equation (corresponding to the switch value) was fixed to the switch value of the first CRP, whereas the other three parameters were treated as free parameters. The AIC goodness-of-fit values from the two cases were compared to determine whether the unconstrained fit was of better quality (lower AIC value) than the constrained fit. If so, the switch value of the second CRP was deemed to be valid, and the resulting shift in the switch value between the two CRPs, significant. If not, the shift in the switch value was deemed to be not significant (indicated in Fig. $5 E$ with light shading). This procedure ensured that the shift in the switch value was significant only if the switch value of one CRP was not sufficient to explain the responses in both of the CRPs.

Instantaneous firing rates (see Fig. 3) were obtained from the peristimulus time histograms (binned at $1 \mathrm{~ms}$ ) by filtering with a Gaussian kernel with $\sigma=12 \mathrm{~ms}$. Pooling was achieved by normalizing the responses to the maximum for each unit and combining across units. This was done separately for units with gradual and switch-like CRPs.

To analyze the time course of suppression by the $S_{\text {out }}$ stimulus, we performed a millisecond-by-millisecond ANOVA, comparing the instantaneous firing rates with $S_{\text {in }}$ presented alone and with $S_{\text {in }}$ and $S_{\text {out }}$ presented together. The time-to-suppression was defined as the first millisecond at which the $p$ value of the ANOVA comparison dropped below 0.05 , remained below 0.05 for the next $25 \mathrm{~ms}$, and reached 0.01 at least once in that period.

The pooled population responses in Figure 6 were obtained by normalizing the responses of a unit ("win" or "lose" condition) by its maximum response and pooling across units (Horwitz and Newsome, 1999; Bisley and Goldberg, 2003) by binning along the relative strength axis ( $x$-axis). The maximum response of a unit was computed as the maximum across responses to both paired and single stimulus conditions. 
Note that all stimulus conditions in Figure $6 \mathrm{~A}$ were randomly interleaved with all the conditions in Figure 6C.

The discriminability between two distributions, $d^{\prime}$, was calculated as $\left(m_{1}-m_{2}\right) / \mathrm{V}\left(s_{1}\right.$ * $\left.s_{2}\right)$, where $m_{1}$ and $m_{2}$ are the means and $s_{1}$ and $s_{2}$ are the SDs of the two sampled distributions. The responses of a unit were normalized (see Figs. 4,5 ) to the maximum response of that unit across both single stimulus and paired stimulus conditions.

Effect of tranquilization. The experiments described in Figure 7 and supplemental Figure S6 (available at www.jneurosci.org as supplemental material) were performed on three birds that remained quiescent when restrained without tranquilization. In these experiments, nitrous oxide tranquilization was turned off after the owl had been positioned and the electrode was at a recording site. Responses to single and paired stimuli were then obtained. For a subset of the units, the measurements were repeated after turning the nitrous oxide on and then repeated a third time after turning the nitrous oxide off. We waited 5-7 min after turning on or off the nitrous oxide before performing the measurements.

We reasoned that 5-7 min was a sufficient waiting period for the action of nitrous oxide and for recovery from it based on the following factors. Nitrous oxide is a low-potency anesthetic [high minimal alveolar concentration (MAC), 104\%] with rapid partitioning (onset and recovery) characteristics (low blood/gas partition coefficient, 0.46 ; low brain/gas partition coefficient, 0.49) (Eger, 2004). In contrast, strong anesthetics such as sevofluorane have a low MAC (MAC of $2 \%$ ) and slow partitioning characteristics (blood/gas partition coefficient, 0.65 ; blood/gas partition coefficient, 1.1) (Eger, 2004). Recovery time from the slow partitioning sevofluorane has been estimated as $\sim 12$ min (Chiu et al., 2000). Furthermore, we have observed that, when unrestrained, an owl stands up and may fly within tens of seconds after nitrous oxide is discontinued.

To test for statistical significance of the effects of tranquilization (see Fig. 7), we estimated the $95 \%$ bootstrap confidence intervals for the mean percentages in the nontranquilized and tranquilized conditions using a standard bootstrap procedure with 1000 resamplings (Efron and Tibshirani, 1994) (bootci function in MATLAB). Overlapping confidence intervals indicated no significant difference at the 0.05 level. The indicated SEM values in the text for Figure 7 were also estimated using the bootstrap procedure.

\section{Results}

Switch-like and gradual suppression by competing stimuli

To study how the strength of a competing stimulus influences the responses of OTi-d units, we presented one stimulus of fixed strength inside the receptive field $\left(\mathrm{S}_{\mathrm{in}}\right)$ and systematically varied the strength of a second, distant stimulus presented $30^{\circ}$ outside the receptive field $\left(\mathrm{S}_{\text {out }}\right)$ (Fig. 1A) (see Materials and Methods). For these experiments, both test stimuli $\left(\mathrm{S}_{\text {in }}\right.$ and $\left.\mathrm{S}_{\text {out }}\right)$ were chosen to be expanding (looming) dots because looming stimuli drive OTi-d units effectively over many repetitions (Mysore et al., 2010) (supplemental Fig. S1 A, B, available at www.jneurosci.org as supplemental material) (see Materials and Methods). The loom speed of $S_{\text {in }}$ (rate of expansion of the dot, its strength) was chosen such that it evoked a strong response (at least $50 \%$ of the maximal response; median speed, $7.6 \%$ ). The loom speed of $\mathrm{S}_{\text {out }}$ was varied from slower to faster than $S_{\text {in }}$ (typical range, $0-22 \%$ ). We recorded unit responses to $S_{\text {in }}$ when it was presented either alone or simultaneously with the $\mathrm{S}_{\text {out }}$ stimulus (Fig. $1 \mathrm{~A}$ ).

Of the units tested in this manner, a majority (107 of 169 , $63 \%$ ) exhibited responses that were negatively correlated with the speed of the $\mathrm{S}_{\text {out }}$ stimulus (Fig. $1 B, C ; p<0.05$, correlation test) (see Materials and Methods). For these units, the maximum response suppression caused by the fastest $\mathrm{S}_{\text {out }}$ speed $\left(22^{\circ} / \mathrm{s}\right)$ decreased responses by an average of $61 \pm 7 \%$ with respect to the responses to $\mathrm{S}_{\text {in }}$ alone (supplemental Fig. $\mathrm{S} 1 \mathrm{~F}$, available at www. jneurosci.org as supplemental material). Of the remaining units, a small fraction (12 of 169, 7\%) showed a fixed response suppression that was independent of the speed of $\mathrm{S}_{\text {out }}$ (see Materials and Methods), whereas the remainder (50 of 169, 30\%) were not affected by the presence of the looming $\mathrm{S}_{\text {out }}$ stimulus (see Materials and Methods). Thus, the responses of a majority of OTi-d units to a stimulus inside the receptive field decreased with increasing strength of a distant, competing stimulus.

Additional analysis of this competitive interaction revealed that, among the units with responses that were negatively corre- 


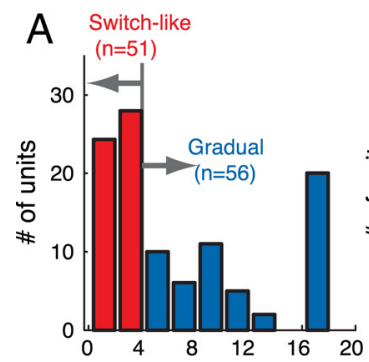

CRP transition range $(\% / s)$

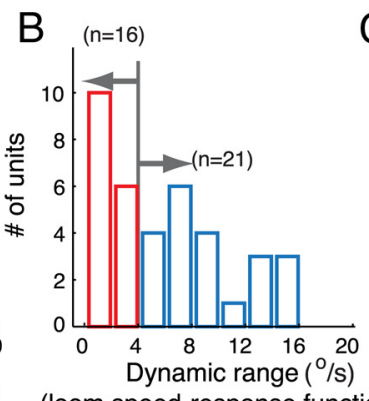

(loom speed-response function)

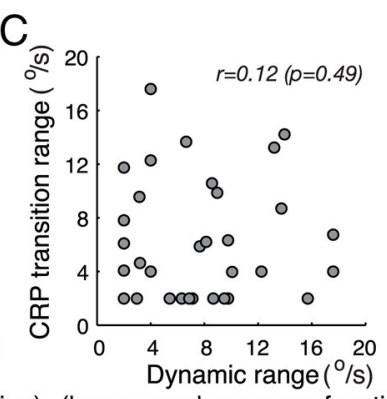

(loom speed-response function)

Figure 2. Population summary of switch-like and gradual responses. $\boldsymbol{A}$, Distribution of transition ranges of CRPs exhibiting a negative correlation with the strength of the looming visual $S_{\text {out }}$ stimulus $(n=107$ of 169). The median strength of the looming $\mathrm{S}_{\text {in }}$ stimulus was $7.6^{\circ} / \mathrm{s}$ with a $95 \%$ confidence interval of $\left[6,9^{\circ} / \mathrm{s}\right]$. $\boldsymbol{B}$, Distribution of dynamic ranges (see Results) of single stimulus $\left(S_{\text {in }}\right)$, loom speed-response functions. $C$, Scatter plot of CRP transition ranges and loom speed-response function dynamic ranges for 37 units showing no correlation between the steepness of the two functions. The datum at $(2,2)$ corresponds to five units, and that at $(4,4)$ corresponds to two units.

lated to the strength of $S_{\text {out }}(n=107)$, the shapes of the response profiles could be very different. We refer to these response profiles as CRPs. Figure $1 B$ shows an example of a unit for which the CRP exhibited gradual response reduction with increasing strength of $\mathrm{S}_{\text {out }}$. In contrast, Figure $1 C$ shows an example of a unit for which the CRP exhibited an abrupt response reduction: a large reduction in responses over a narrow range of $S_{\text {out }}$ speeds.

To quantify the abruptness of such response transitions, we defined as the CRP "transition range," the range of $\mathrm{S}_{\text {out }}$ loom speeds over which responses dropped from 90 to $10 \%$ of the total range of responses (see Materials and Methods) (supplemental Fig. S1G, available at www.jneurosci.org as supplemental material). The range of responses was estimated from either the best sigmoidal fit or the best linear fit to the CRP data, depending on which yielded a better goodness-of-fit (see Materials and Methods). The abruptly changing responses shown in Figure $1 C$ were best fit by a sigmoidal function $\left(r^{2}=0.96\right)$ and had a narrow transition range of $0.4^{\circ} \mathrm{s}$. In contrast, the gradually changing responses in Figure $1 \mathrm{~B}$, also best fit by a sigmoidal function $\left(r^{2}=\right.$ $0.95)$, had a large transition range of $7.5^{\circ} \mathrm{s}$. In addition, some units exhibited gradually changing responses that were best fit by a linear function (Fig. 1D). For these units, the transition range spanned nearly the entire range of speeds tested (by definition, all linear CRPs yielded the same transition range, equal to $80 \%$ of the tested range of loom speeds).

The distribution of transition ranges across the entire population of tested units (Fig. $2 A$ ) revealed a significant fraction of units that had very narrow transition ranges (left end of the distribution), the rest having intermediate (middle of the distribution) or large transition ranges (right end of the distribution, corresponding to CRPs best fit by a linear function). In constructing this distribution, when the calculated transition range of a CRP was less than the sampling increment, the transition range was rounded up to the value of the sampling increment. This procedure resulted in a conservative estimate of the transition ranges of abruptly changing responses. For instance, for the CRP shown in Figure $1 C$, the calculated transition range of $0.4^{\circ} \mathrm{s}$ was rounded up to the sampling increment of $2.4 \%$. The median sampling increment was $3.2 \pm 0.17 \%$ s, and the largest was $4 \%$ s. The distribution of transition ranges using the non-rounded-up values is shown in supplemental Figure S2 (available at www. jneurosci.org as supplemental material).

To explore the implications of abrupt response transitions to the representation of relative stimulus strength, we focused our
$[0.82,0.90]$ for switch-like CRPs), and the quality of the fits was not significantly different between the two types of CRPs ( $p=$ 0.28 , rank-sum test).

We wondered whether the abruptness of the CRP was related to the abruptness of the single stimulus-response function. To address this question, we measured loom speed-response functions by systematically varying the loom speed of a single stimulus $\left(S_{\text {in }}\right)$ centered in the receptive field. This was done for a subset of 56 units for which CRPs were also measured. The range of loom speeds tested was $0-22 \%$. For all tested units, the responses were positively correlated with the strength of the $\mathrm{S}_{\text {in }}$ stimulus $(p<$ 0.05 , correlation test). To characterize the abruptness of loom speed-response functions, we defined their "dynamic range" as the range of $S_{\text {in }}$ loom speeds over which the responses changed from 10 to $90 \%$ of the total range of responses (supplemental Fig. $\mathrm{S} 2 B$, available at www.jneurosci.org as supplemental material). The distribution of dynamic ranges is shown in Figure $2 \mathrm{~B}$. As with CRPs, we characterized loom speed-response functions with dynamic ranges $\leq 4 \%$ s as being switch-like and the remaining as being gradual.

We compared these loom speed-response functions with CRPs measured from the same units. Of the 56 units for which both measurements were performed, CRPs were negatively correlated with the strength of $S_{\text {out }}$ for 37 units. The scatter plot of CRP transition ranges versus loom speed dynamic ranges for these units showed no systematic relationship (Fig. $2 C$; correlation coefficient $=0.12, p=0.49)$. In addition, there was no significant difference between the distributions of dynamic ranges for units with switch-like or gradual CRPs (mean dynamic ranges: gradual, $7 \pm 1.1 \%$; switch-like, $7.1 \pm$ $1.1 \%$; $p=1, t$ test). Thus, single stimulus-response functions were not predictive of the abruptness of competitive response transitions in the OTi-d.

We also tested whether the nature of the CRP (switch-like vs gradual) was systematically related to receptive field location, $S_{\text {out }}$ stimulus location, or receptive field size and found no systematic relationship with any of these parameters (supplemental Fig. S3, available at www.jneurosci.org as supplemental material). Thus, switch-like or gradual response modulation by a competing stimulus does not depend on the spatial properties of the receptive field or the specific location of the competing stimulus. 

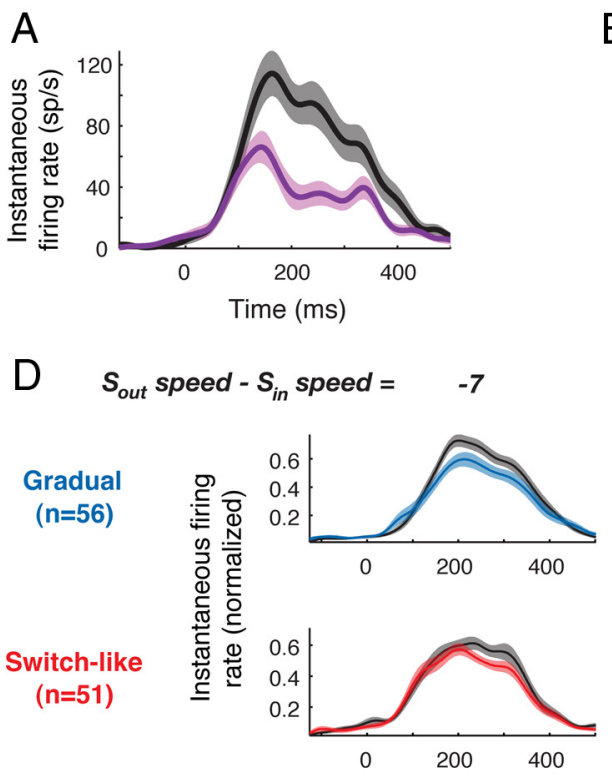
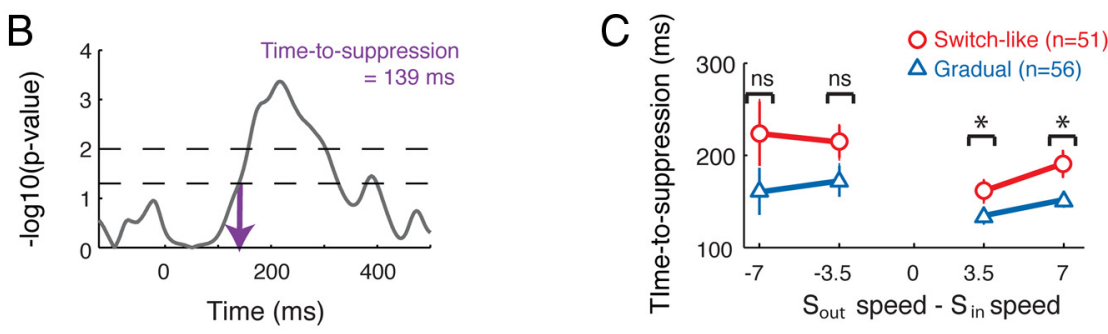

$-3.5$
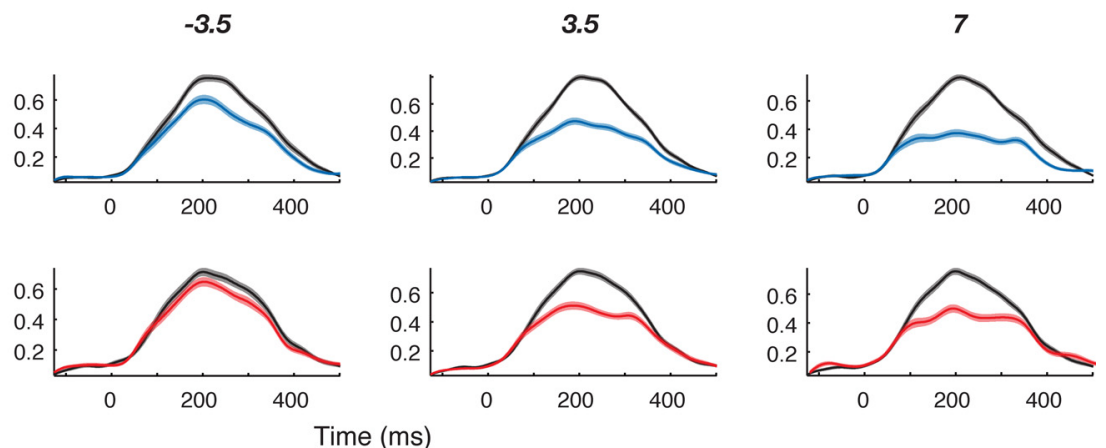

Figure 3. Time course of response suppression for units with switch-like and gradual responses. $A$, Average instantaneous firing rate responses of an 0 Ti-d neuron to $S_{\text {in }}$ presented alone (in black) or to $S_{\text {in }}$ and $S_{\text {out }}$ presented together (in purple). Line, Mean; shading, SEM. $S_{\text {in }}$ loom speed, $10 \%$; $S_{\text {out }}$ loom speed, $13.5 \%$ s. $B, p$ value from a millisecond-by-millisecond ANOVA comparing the instantaneous firing rates in $A$. Dashed lines indicate $p=0.05$ and $p=0.01$ levels. The time-to-suppression was defined as the first millisecond at which the $p$ value of the ANOVA comparison dropped below 0.05 , remained below 0.05 for the next $25 \mathrm{~ms}$, and reached 0.01 at least once in that period. $C$, Population average of time-to-suppression as a function of the difference between $S_{\text {out }}$ and $\mathrm{S}_{\text {in }}$ strength, grouped into four bins. Median $\mathrm{S}_{\text {in }}$ strength of $7.6 \%$ for units with both gradual and switch-like CRPs. ${ }^{*}$ indicates significance at the 0.05 level (paired $t$ tests with Holm-Bonferroni correction). $\boldsymbol{D}$, Pooled averages of the instantaneous firing rate responses binned as in $C . S_{\text {out }}-S_{\text {in }}$ strength indicated above the panels. Black, Responses to $S_{\text {in }}$ alone; color, responses to $S_{\text {in }}$ and $S_{\text {out }}$ together. $S_{\text {in }}$ and $S_{\text {out }}$ were presented between 0 and $250 \mathrm{~ms}$.

\section{Time course of response suppression for units with switch-like versus gradual CRPs}

Units with gradual and switch-like CRPs exhibited different time courses of response suppression. We calculated the instantaneous firing rate responses (see Materials and Methods) to $S_{\text {in }}$ alone and to $S_{\text {in }}$ and $S_{\text {out }}$ presented simultaneously (Fig. $3 A$ ) and compared these rates using a millisecond-by-millisecond ANOVA procedure (Fig. $3 B$ ). The time of emergence of response suppression ("time-to-suppression") was defined as the first time point at which the firing rates diverged significantly (see Materials and Methods). We repeated this procedure for each unit, for each value of the competitor strength in the CRP, and we calculated the time-to-suppression as a function of the difference between $\mathrm{S}_{\text {out }}$ and $\mathrm{S}_{\text {in }}$ strengths (see Materials and Methods).

Population analysis demonstrated that time-to-suppression was another metric of competition that distinguished the responses of units with switch-like versus gradual CRPs (Fig. 3C). Although no difference was found in onset latencies to the $S_{\text {in }}$ stimulus alone (gradual, latency of $115 \pm 11 \mathrm{~ms}$; switch-like, latency of $107 \pm 11 \mathrm{~ms} ; p=0.56, t$ test), in the context of stimulus competition, the time-to-suppression was significantly longer for units with switch-like CRPs when $\mathrm{S}_{\text {out }}$ was the stronger stimulus (Fig. $3 C$, right side, circles vs triangles; $p<0.05$, paired $t$ tests with Holm-Bonferroni correction). When $S_{\text {out }}$ was the weaker stimulus, the time-to-suppression was nominally (but not significantly) longer for units with switch-like CRPs (Fig. 3C, left side; $p>0.05)$. In addition, for both groups of units, the average time-to-suppression was significantly shorter when $S_{\text {out }}$ was the stronger stimulus (gradual, $141 \pm 6$ vs $169 \pm 14 \mathrm{~ms}, p=0.03, t$ test; switch like, $172 \pm 9$ vs $217 \pm 16 \mathrm{~ms}, p=0.013, t$ test).

Population averages of the instantaneous firing rates across units demonstrated the same effects (Fig. 3D).
Sensory modality independence of switch-like versus gradual suppression by a competing stimulus

Next, we investigated whether gradual and switch-like modulations of responses occurred independently of the nature of the competing stimulus. To address this question, we measured CRPs using an $\mathrm{S}_{\text {out }}$ stimulus of a different sensory modality. The alternative $S_{\text {out }}$ stimuli we used were noise bursts of different sound levels; the $\mathrm{S}_{\text {in }}$ stimulus was the same looming visual stimulus as before (Fig. 4A). Of the units tested in this manner, a majority (40 of 66, 61\%) exhibited CRPs that were negatively correlated with the strength of the auditory $\mathrm{S}_{\text {out }}$ stimulus $(p<$ 0.05 , correlation test). Figure $4 B$ shows an example of one such unit, for which responses to the paired stimuli changed abruptly as the strength of $\mathrm{S}_{\text {out }}$ was increased. For these units, the maximum response suppression at $50 \mathrm{~dB}$ above threshold was $-23 \pm$ 7.6\% (supplemental Fig. S4, available at www.jneurosci.org as supplemental material). Of the remaining units, a small fraction showed a fixed response suppression that was independent of the strength of $\mathrm{S}_{\text {out }}$ ( 2 of $66,3 \%$ ), whereas the remainder ( 24 of 66 , $36 \%$ ) were unaffected by the presence of this particular auditory $\mathrm{S}_{\text {out }}$ stimulus.

For the CRPs that were negatively correlated with the strength of $\mathrm{S}_{\text {out }}(n=40)$, we obtained the best-fitting function, between sigmoidal and linear functions, and calculated the CRP transition ranges. As before, when the calculated transition range was smaller than the sampling increment, it was rounded up to the sampling increment. The median sampling increment was $8.6 \mathrm{~dB}$ (with a $95 \%$ confidence interval of $[6,10 \mathrm{~dB}]$ ), and the largest was $10 \mathrm{~dB}$. The distribution of transition ranges across these units revealed that a large fraction of CRPs had narrow transition ranges (Fig. 4C). CRPs were considered to be switch-like if the transition range was $\leq 10 \mathrm{~dB}$ and gradual otherwise. The cutoff of 

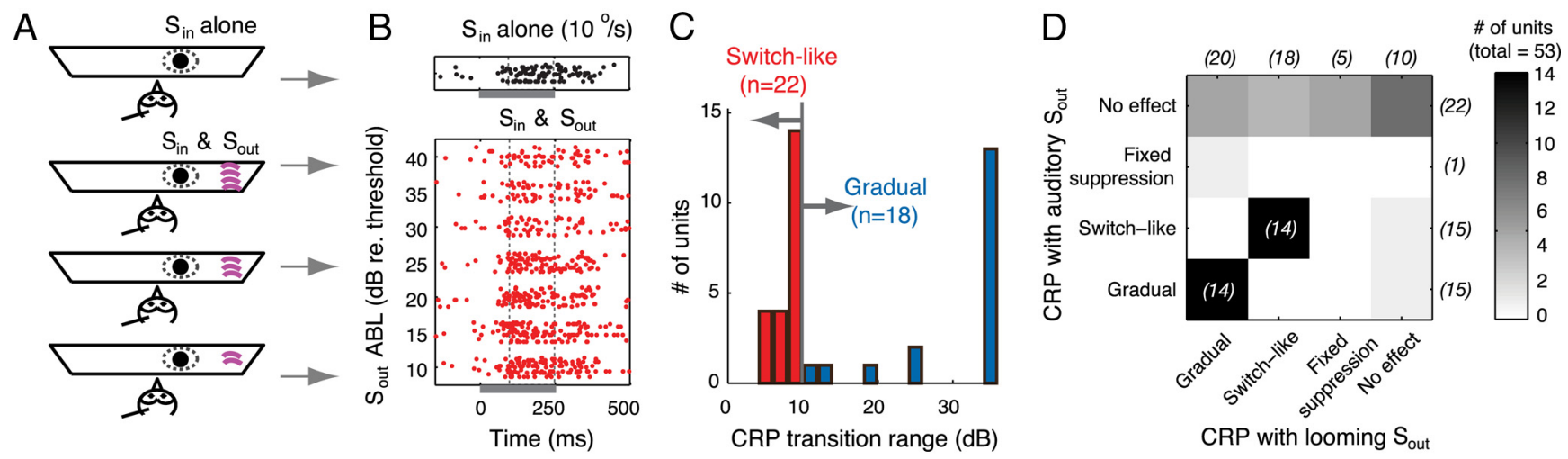

Figure 4. Sensory modality independence of switch-like versus gradual suppression by a competing stimulus. $A$, Schematic of the experimental protocol with $S_{\text {in }}$ being a looming visual stimulus of fixed strength and $S_{\text {out }}$ being a broadband noise burst of different levels. $\boldsymbol{B}$, Rasters of switch-like unit responses (conventions as in Fig. $1 B, C$ ). Correlation coefficient of the firing rates (100 - 250 ms time window) versus $\mathrm{S}_{\text {out }}$ strength was -0.86 ( $p=0.002$, Spearman's correlation test). Calculated transition range of $2.2 \mathrm{~dB}$. C, Distribution of transition ranges of (RPs exhibiting a negative correlation with the strength of the auditory $S_{\text {out }}$ stimulus $(n=40$ of 66 ); a large fraction of units exhibited CRPs with narrow transition ranges (left end of distribution). CRPs were considered to be switch-like if their transition range was $\leq 10 \mathrm{~dB}$ (indicated in red; see Results) and gradual (indicated in blue) otherwise. The median strength of the looming visual $\mathrm{S}_{\text {in }}$ stimulus was $6.4 \%$ with a $95 \%$ confidence interval of $[6.4,9.6 \%$. $]$. Delationship between the type of CRP (gradual, switch-like, fixed suppression, no effect) measured with a looming visual $S_{\text {out }}$ stimulus and that of the CRP measured with an auditory $S_{\text {out }}$ stimulus, when both CRPs were measured in an interleaved manner ( $n=53$ units). Numbers in parentheses outside the correlation matrix represent row or column totals, as appropriate.

$10 \mathrm{~dB}$ represented no more than two sampling increments, and was one-fifth of the total range of tested sound levels (see Materials and Methods). Based on this criterion, 55\% (22 of 40) of the CRPs were switch-like (Fig. 4C), whereas the rest (18 of 40) were gradual. Thus, stimulus competition across sensory modalities also yielded switch-like responses.

We next asked whether the type of CRP (switch-like, gradual, fixed suppression, or no-effect) measured using an $\mathrm{S}_{\text {out }}$ stimulus of one sensory modality was predictive of the type of CRP measured with an $\mathrm{S}_{\text {out }}$ stimulus of a different sensory modality. To address this question, we measured, for a subset of units $(n=53)$, CRPs with two different $S_{\text {out }}$ stimuli: a looming visual $S_{\text {out }}$ stimulus and a noise burst auditory $S_{\text {out }}$ stimulus. The $S_{\text {in }}$ stimulus was a looming visual stimulus, and the two CRPs were measured in an interleaved manner.

As summarized in Figure $4 D$, of the 20 units for which the CRP measured with the looming $\mathrm{S}_{\text {out }}$ stimulus was gradual, a majority (14 of 20) also exhibited a gradual CRP with the auditory $\mathrm{S}_{\text {out }}$ stimulus; the remaining units showed either no effect of this auditory $S_{\text {out }}$ stimulus ( 5 of 20) or a fixed suppression ( 1 of 20). Similarly, of the 18 units for which the CRP measured with looming $S_{\text {out }}$ stimuli was switch-like, a majority (14 of 18) also exhibited a switch-like CRP with the auditory $\mathrm{S}_{\text {out }}$ stimuli; the remaining units showed no effect of this auditory $\mathrm{S}_{\text {out }}$ stimulus (4 of 18). Conversely, nearly all units that exhibited gradual (15) or switch-like (15) CRPs with auditory $\mathrm{S}_{\text {out }}$ stimuli exhibited, respectively, gradual (14 of 15) and switch-like (14 of 15) CRPs with looming $\mathrm{S}_{\text {out }}$ stimuli. Thus, gradual and switch-like modulations of unit responses by a competing stimulus occurred essentially independently of the nature of the competing stimulus.

\section{Dependence of switch value on the strength of the $\mathrm{S}_{\text {in }}$ stimulus}

The abruptness of the transition in switch-like CRPs indicated that the strength of the competitor that caused this transition was well defined. We called this competitor strength the switch value and estimated it as the midpoint of the transition range of a switch-like CRP (see Materials and Methods). This led us to ask the following: how does the switch value relate to the strength of the stimulus inside the receptive field? The switch value of the switch-like CRP in Figure 1C2 was $7.2^{\circ} / \mathrm{s}$, nearly equal to the constant strength of $\mathrm{S}_{\text {in }}(8 \%)$ (Fig. $\left.5 A\right)$. To test whether this equality held true across the population, we plotted the distribution of the differences between the switch value and the $S_{\text {in }}$ strength for 51 switch-like CRPs (Fig. 5B). The mean difference was not significantly different from $0\left(0.92 \pm 0.66^{\circ} / \mathrm{s}, p=0.12, t\right.$ test). Thus, across the population of switch-like CRPs, responses transitioned from high to low values when the strength of the competitor was nearly equal to the strength of the $S_{\text {in }}$ stimulus.

This observation suggested the interesting possibility that the switch value of a switch-like CRP may not be fixed but instead may depend specifically on the strength of the stimulus inside the receptive field. Furthermore, the observation that the switch value can be greater or smaller than the strength of $S_{\text {in }}$ (indicated by the spread of the distribution in Fig. $5 B$ ) suggested that this dependence of CRP switch value on $S_{\text {in }}$ strength may be a proportionality rather than an equality. If true, these hypotheses predict that changing the strength of the $S_{\text {in }}$ stimulus should shift the switch value of the CRP predictably. To test these hypotheses, we measured CRPs with $S_{\text {in }}$ stimuli of two different strengths for a subset of units that exhibited switch-like responses (Fig. 5C;n= 16 units). Both $S_{\text {in }}$ and $S_{\text {out }}$ were looming visual stimuli in these tests, and all stimuli were interleaved.

Figure 5D shows an example of switch-like CRPs (and the corresponding best fits) from one unit, measured with $S_{\text {in }}$ loom speeds of 4 and $10 \%$, respectively. In addition to the increase in the overall firing rate of the unit (consistent with an increase in the excitatory drive from the faster $S_{\text {in }}$ stimulus), the switch value of the CRP shifted rightward with an increase in $S_{\text {in }}$ strength (Fig. $5 D$, horizontal arrow). For this unit, the switch values of the two CRPs were, respectively, 1.9 and $7.8 \%$ s, and the resulting shift in switch value was $5.9 \%$, nearly equal to the $6 \%$ change in the strength of $S_{\text {in }}$. The validity of the shift in the switch value was tested by verifying that no sigmoid with the same switch value as the first CRP $\left(1.9^{\circ} / \mathrm{s}\right)$ was as good as the best-fitting sigmoid to the second CRP (Fig. 5D, green curve) (see Materials and Methods).

Predictable shifts in CRP switch values in response to changing the strength of $S_{\text {in }}$ were observed consistently across a population of 16 units exhibiting switch-like CRPs. Figure $5 E$ shows the distribution of shift ratios, the ratio of the shift in switch value 
to the change in the strength of $S_{\text {in }}$, across the population. The mean ratio was $0.90 \pm 0.16$ and was not significantly different from 1 ( $p=0.55, t$ test). The mean shift ratio for gradual CRPs that were best fit by a sigmoid was not significantly different from that for switch-like CRPs (supplemental Fig. S5C, available at www. jneurosci.org as supplemental material; $0.57 \pm 0.09, n=12 ; p=0.11$, $t$ test against distribution in Fig. 5E). Thus, the switchvalues of switch-like CRPs depended specifically on the strength of the stimulus inside the receptive field. This indicated that units with switch-like CRPs acted like two-state comparators of the strength of the reference stimulus inside the receptive field relative to the strength of a competing stimulus outside the receptive field.

\section{Signaling the strongest stimulus across the OTi-d space map: switch-like versus gradual responses}

The results so far suggest that switch-like responses may signal the stronger of two competing stimuli across the OTi-d space map. However, the absolute firing rate of a unit did not, by itself, indicate the stronger stimulus unambiguously. This is exemplified by the switch-like responses shown in Figure 5D. For this unit, a firing rate of 100 spikes/s occurred both when the $S_{\text {in }}$ stimulus was stronger than $S_{\text {out }}\left(S_{\text {in }}\right.$ speed, $4 \%$ ) (Fig. $5 D$, purple curve, left side) and when the $S_{\text {in }}$ stimulus was weaker than $\mathrm{S}_{\text {out }}\left(\mathrm{S}_{\text {in }}\right.$ speed, $10 \%$ ) (Fig. $5 D$, green curve, right side). The overall increase in firing rates produced by the increase in the strength of $S_{\text {in }}$ was the source of this ambiguity, and such an increase was observed consistently across the 16 units tested that exhibited switch-like responses (supplemental Fig. S5D, available at www.jneurosci.org as supplemental material).

How, then, does the OTi-d signal the stronger of competing stimuli across the OTi-d space map? To address this question, we compared unit responses with the simultaneous presentation of paired $\left(S_{\text {in }}\right.$ and $\left.S_{\text {out }}\right)$ stimuli of unequal strengths, measured in two mirror-symmetric conditions (Fig. 6A). In one condition, called the "win" condition, the stronger stimulus was presented inside the receptive field and the weaker one outside $\left(S_{\text {in }}>S_{\text {out }}\right)$. In the second condition, the "lose" condition, the stimulus strengths were reversed. To analyze the coding of the stronger stimulus, the responses in the win condition were compared with the responses in the corresponding lose condition, pooled across all units (Horwitz and Newsome, 1999; Bisley and Goldberg, 2003). The pooled responses from the lose condition served as a surrogate for the responses in the OTi-d space map encoding the location of the $S_{\text {out }}$ stimulus. This approach assumes that unit populations at the two locations encoding $S_{\text {in }}$ and $S_{\text {out }}$, respectively, have similar functional properties.

To study the effect of changes in relative stimulus strength, the magnitude of the difference between $S_{\text {in }}$ and $S_{\text {out }}$ was systematically varied in these tests (Fig. $6 \mathrm{~A}$ ). Both $\mathrm{S}_{\text {in }}$ and $\mathrm{S}_{\text {out }}$ were chosen to be looming visual dots, and all conditions were randomly in-
C
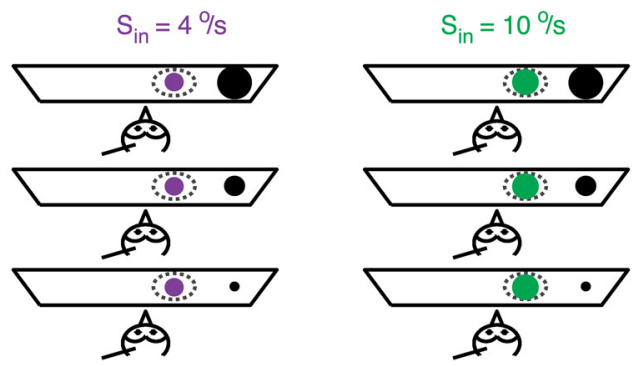

D

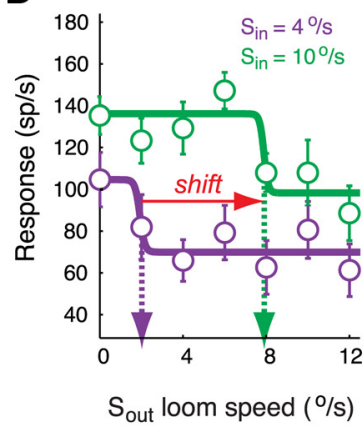

E

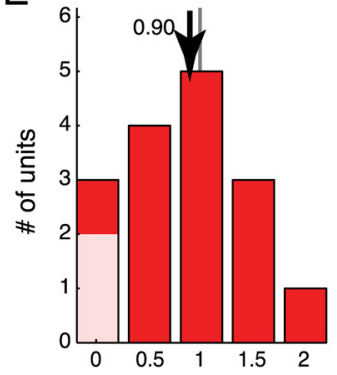

Shift in switch-value

Change in strength of $S_{\text {in }}$

Figure 5. Dependence of switch value on the strength of the $S_{\text {in }}$ stimulus. $A$, Definition of switch value for the switch-like CRP in Figure (see Materials and Methods). $S_{\text {in }}, 8 \%$; switch value, $7.2 \%$. B , Distribution of (switch value - strength of $S_{\text {in }}$ ) for significantly different from 1 ( $p=0.55, t$ test against 1). Median strength of $S_{\text {in }}$ for the first CRP was $6 \%$ (with a $95 \%$ confidence interval of $[5.4,6 \% \mathrm{~s}])$, and the change in the strength of $S_{\text {in }}$ was $6 \%$.

terleaved. These tests were performed on a subset of the units described in Figures 1 and 2 (62 of 169), of which 20 showed gradual CRPs and 13 showed switch-like CRPs; the remaining units either showed fixed response suppression $(n=6)$ or no suppression by the $\mathrm{S}_{\text {out }}$ stimulus $(n=23)$. The pooled population responses (see Materials and Methods) of units exhibiting gradual (Fig. $6 B$, left) and switch-like (Fig. $6 B$, right) CRPs are shown for the win and lose conditions, as a function of relative stimulus strength. For each pair of win versus lose conditions, we compared the responses using discriminability analysis with the $d^{\prime}$ metric (see Materials and Methods). This analysis quantifies the ability of an ideal observer to correctly discriminate the stronger stimulus based solely on the responses at the two locations in the OTi-d space map; larger $d^{\prime}$ values indicate greater discriminability.

For units with gradual CRPs, discriminability increased systematically with the difference in the strengths of the competing stimuli (Fig. $6 E$, left, data in purple; $p=0.02$, correlation test). In contrast, for units with switch-like CRPs, discriminability was high even for small values of relative stimulus strength and remained high for larger values (Fig. $6 E$, right, data in purple; $p=$ 0.38 , correlation test). As a result, when the difference in stimulus strengths was small, the ability to signal the stronger stimulus was substantially greater for units with switch-like than gradual CRPs (Fig. $6 E$, left vs right, purple squares; $d^{\prime}$ at $\Delta=2 \%$ was $0.8 \pm 0.18$ for units with gradual CRPs and $2.48 \pm 0.38$ for units with switch-like CRPs; $p=0.007, t$ test). For large differences in stim- 
A
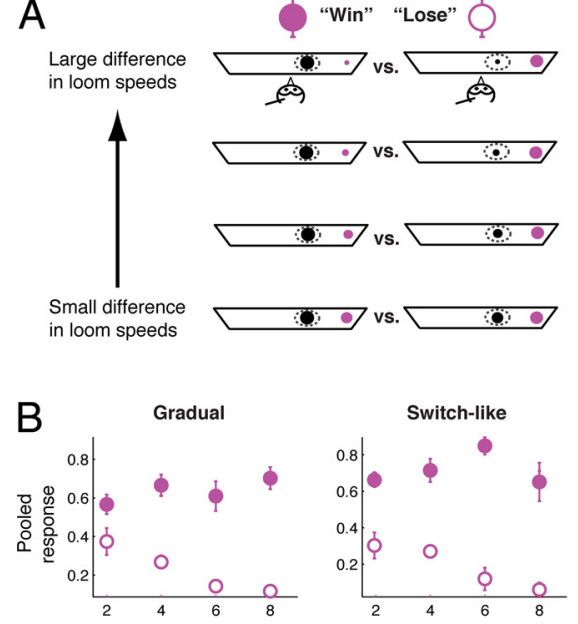

$E$

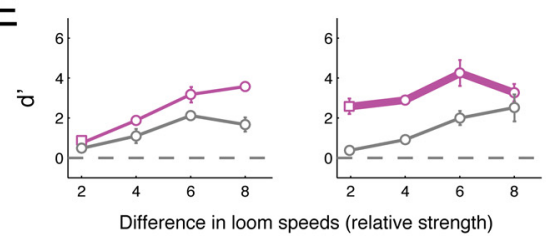

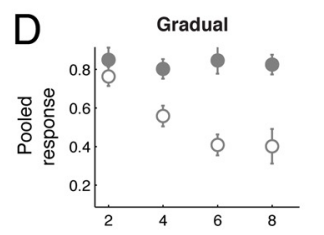

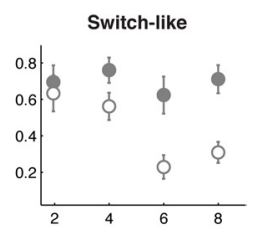

C

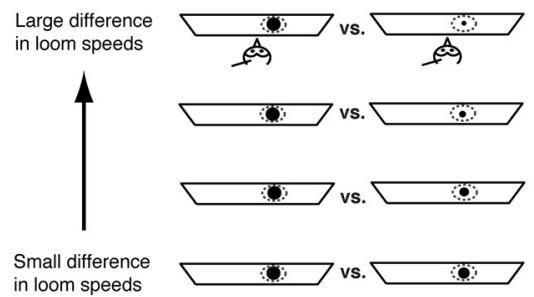

$\mathrm{F}$

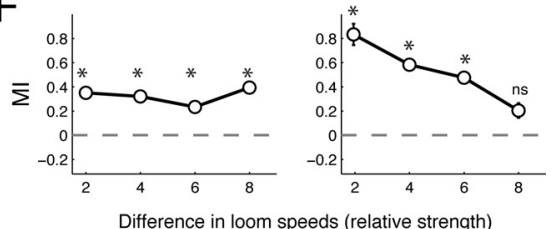

Figure 6. Signaling the stronger stimulus across the 0Ti-d space map. $A$, Experimental protocol. Pairs of $S_{\text {in }}$ and $S_{\text {out }}$ stimuli of unequal strengths are presented in two stimulus conditions: win, with the stronger stimulus inside the receptive field; and lose, with the weaker one inside. Such tests were performed at different values of relative stimulus strength achieved by fixing the strength of the stronger stimulus and systematically varying the strength of the weaker one; $S_{\text {in }}$ and $S_{\text {out }}$ were looming visual dots. For instance, when the strength of the stronger stimulus was chosen to be $9 \%$, the win conditions corresponding to the different relative strengths of $2,4,6$, and $8 \%$ s were $(9$, $7 \% / 5),\left(9,5^{\circ} / \mathrm{s}\right),\left(9,3^{\circ} / \mathrm{s}\right)$, and $\left(9,1^{\circ} / \mathrm{s}\right)$, respectively; pairs indicate $\mathrm{S}_{\text {in }}$ and $\mathrm{S}_{\text {out }}$ strengths. The fixed strength of the stronger stimulus was chosen such that it evoked at least $50 \%$ of the maximal response for that unit (same as the criterion used for choosing $S_{\text {in }}$ strength in Fig. 1). $\boldsymbol{B}$, Pooled responses (see Materials and Methods) in the win (filled purple circles) and lose (open purple circles) conditions from 20 units with gradual CRPs (left) and 13 units with switch-like CRPs (right). The average strength of the stronger stimulus was $8 \%$ s for both types of CRPs. C, Experimental protocol for measurements of responses to $S_{\text {in }}$ alone. Win and lose conditions weretested in the absence of the $S_{\text {out }}$ stimulus randomly interleaved with the tests in $A$. Each row represents the stimulus condition that tests the condition in the corresponding row in $A$ but in the absence of $S_{\text {out }} \cdot \boldsymbol{D}$, Pooled responses in the win (filled gray circles) and lose (open gray circles) conditions from the same units with gradual (left, $n=20$ ) and switch-like (right, $n=13$ ) CRPs, as in A; obtained using the same procedure. E, Discriminability ( $d^{\prime}$; see Materials and Methods) of the stronger stimulus as a function of the relative stimulus strength for units with gradual (left) and switch-like (right) CRPs. Purple, Data from protocol in $\boldsymbol{A}$; gray, data from protocol in $\boldsymbol{C}$. Thick line in right denotes the binary discrimination signal. SEM was estimated by a standard jackknife procedure (Efron and Tibshirani, 1994) and was, in some cases, smaller than the size of the symbol used to indicate the mean. $\boldsymbol{F}, \mathrm{Ml}$ (see Materials and Methods) quantifying the change in discriminability $\left(d^{\prime}\right)$ of the stronger stimulus attributable to inhibitory competitive interactions. Left, Units with gradual CRPs; right, units with switch-like CRPs. Dashed line shows MI $=0$. Data show mean \pm SEM; SEM estimated by a standard bootstrap procedure (Efron and Tibshirani, 1994) with 1000 resamplings. In some cases, the SEM is smaller than the size of the symbol used to indicate the mean. * indicates significance at the 0.05 level ( $t$ tests with HolmBonferroni correction).

ulus strengths, responses of units with both types of CRPs yielded reliable discrimination of the stronger stimulus.

Thus, the relative rates of unit discharges across the OTi-d space map signaled unambiguously the location of the stronger stimulus. In addition, these data reveal that the responses of units with switch-like CRPs yields a binary discrimination signal (Fig. $6 E$, right, thick purple line) that specifically amplifies the representation of the stronger stimulus when the difference in stimulus strengths is small, and it is independent of the magnitude of relative stimulus strength.

\section{Competitive interactions enhance discriminability}

\section{differentially for switch-like versus gradual responses}

The representations of relative strength described thus far involve competitive interactions between the stimuli. However, even without competitive interactions, the systematic relationship that exists between the strength of a stimulus inside the receptive field and unit response rates (single stimulus-response functions) (supple- mental Fig. S1A-E, available at www. jneurosci.org as supplemental material) would lead to a default representation of relative stimulus strength across the space map. What benefits, if any, do competitive interactions provide over such a default representation of relative stimulus strength? To address this question, we compared win and lose responses measured in the presence (Fig. 6A) and absence (Fig. 6C) of the $\mathrm{S}_{\text {out }}$ stimulus. Responses to the $S_{\text {in }}$ stimulus presented alone (Fig. $6 \mathrm{C}$ ) are equivalent to the responses to $S_{i n}$ in the absence of competitive interactions with the $S_{\text {out }}$ stimulus. These experiments were performed on the same units represented in Figure 6, $A$ and $B$, and all conditions were randomly interleaved with those described in Figure 6A.

The pooled population responses to the win (filled circles) and lose (open circles) conditions without competitive interactions (Fig. 6D) are shown on the same axis of relative strength as in Figure $6 B$. As before, we computed the discriminability $\left(d^{\prime}\right)$ between the win and lose conditions, for each value of relative strength (Fig. 6E, left, data in gray, units with gradual CRPs; right, data in gray, units with switch-like CRPs).

The effect of the $\mathrm{S}_{\text {out }}$ stimulus on the discriminability of the strongest stimulus across space was quantified using a modulation index (MI), defined as the difference in discriminabilities in the presence (Fig. $6 E$, data in purple) and absence (Fig. $6 E$, data in gray) of the $S_{\text {out }}$ stimulus, divided by their sum. A positive value of the modulation index indicates an increase in discriminability attributable to the presence of the $S_{\text {out }}$ stimulus, whereas a negative value indicates a decrease. The modulation index revealed that, for units with gradual CRPs, the presence of the $S_{\text {out }}$ stimulus improved the discriminability of the stronger stimulus by the same, constant amount for all relative stimulus strengths (Fig. $6 F$, left; $p=0.92$, correlation test). In contrast, for units with switch-like CRPs, the presence of the $S_{\text {out }}$ stimulus enhanced the discriminability of the stronger stimulus preferentially when the difference between stimulus strengths was small (Fig. $6 \mathrm{~F}$, right), and the contribution of competitive interactions progressively decreased as the difference between stimulus strengths increased ( $p=0.01$, correlation test). Interestingly, in the absence of the $S_{\text {out }}$ stimulus, the discriminabilities of units with switch-like CRPs were indistinguishable from those of units with gradual CRPs (Fig. $6 E$, left vs right panels, data in gray; $p>0.05$, paired $t$ tests with Holm-Bonferroni correction).

Thus, competitive interactions improved the ability of both gradual and switch-like responses to signal the stronger stimulus. Furthermore, competitive interactions were entirely responsible for the enhanced discriminability of the stronger stimulus pro- 


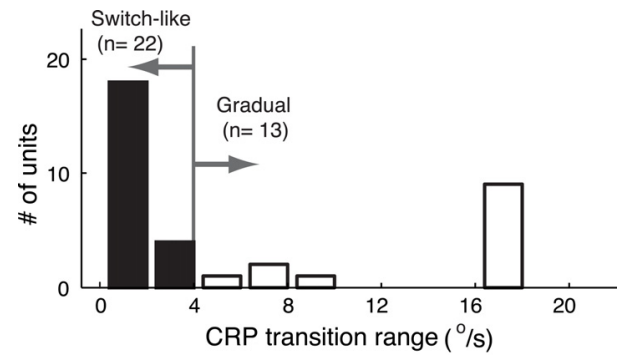

Figure 7. Competitive interactions in nontranquilized animals. Distribution of CRP transition ranges for units for which responses were correlated with the strength of $S_{\text {out }}$ measured in nontranquilized animals. Transition ranges were rounded up to the loom speed sampling increment if they were smaller than the sampling increment.

vided by switch-like responses when differences in stimulus strength were small.

\section{Switch-like CRPs in nontranquilized animals}

Finally, we asked whether the competitive interactions observed in tranquilized owls, presented above, are similar in nontranquilized owls. To address this question, we measured CRPs in nontranquilized owls (see Materials and Methods). Both $S_{\text {in }}$ and $S_{\text {out }}$ stimuli were chosen to be looming visual dots. Of the 49 CRPs measured, $71 \%$ (35 of 49 ) showed a significant negative correlation of the responses to the paired stimuli with the strength of the $\mathrm{S}_{\text {out }}$ competitor compared with $63 \%$ in tranquilized owls (as stated previously); the percentage of correlated CRPs was not significantly different between tranquilized and nontranquilized owls ( $71 \pm 6.3$ vs $63 \pm 3.6 \%$; $p>0.05$ ) (see Materials and Methods). The remaining CRPs (14 of 49) exhibited responses that were uncorrelated with $S_{\text {out }}$ strength.

The distribution of transition ranges for the correlated CRPs is shown in Figure 7. Using the same criterion as in Figure 2, we found that $63 \%$ ( 22 of 35 ) of the correlated CRPs were switch-like compared with $48 \%$ in tranquilized animals (from Fig. 2); the percentage of switch-like CRPs was nominally, but not significantly, larger in nontranquilized owls ( $63 \pm 7.9$ vs $48 \pm 4.9 \%$; $p>$ 0.05 ) (see Materials and Methods). In addition, for a subset of units (36 of 49), we measured responses before, during, and after tranquilization (supplemental Fig. S6, available at www. jneurosci.org as supplemental material) (see Materials and Methods). Competitive interactions in nontranquilized and tranquilized owls were essentially indistinguishable, with the exception of response variability to paired stimuli (supplemental Fig. S6E, average Fano factor, available at www. jneurosci.org as supplemental material), which was lower in nontranquilized owls.

\section{Discussion}

The selection of the next target for spatial attention or gaze is influenced by both the internal goals of the animal (top-down influences) and the physical properties of the stimuli in the world (bottom-up influences). Typically, bottom-up information contributes to target selection but in certain situations can dominate it (Knudsen, 2011). Comparing the properties of multiple stimuli in a complex environment and detecting the most important stimulus is, therefore, an essential component of target selection. Although the OT is known to be an important node in the network of brain areas involved in target selection, the steps by which the representations of competing stimuli are transformed to yield the selection of the next target have remained unclear.
This study reveals a previously unknown and critical step in this transformation.

We examined how the OT represents the relative strengths of simultaneously occurring stimuli that are competing in a bottom-up manner. We interpret the results in the context of sensory computations. Although the responses we observed could well lead eventually to motor-related activity (Wurtz and Goldberg, 1972), it is unlikely that they represent motor plans themselves, because they are reliably stimulus locked to frequent and interleaved stimuli, even in untrained, tranquilized animals. However, it is likely that these responses play a fundamental role in determining spatial goals of motor plans. The location of the strongest stimulus is encoded in the relative firing rates of neurons across the OTi-d, a representation that is similar to the representation of stimulus salience by neurons in the LIP of monkeys (Bisley and Goldberg, 2003). In the owl OTi-d, this is accomplished by neurons that encode relative stimulus strength in a switch-like manner in addition to neurons that do so in a gradual manner.

It is possible that gradual and switch-like responses represent ends of a continuum. However, gradual and switch-like responses exhibited distinct signatures in rate, time course, and stimulus discriminability, suggesting that they may actually correspond to functionally distinct subsets of OTi-d neurons.

The representation of relative stimulus strengths by gradual responses alone has the hallmarks of a map of bottom-up salience, proposed in computational models as subserving bottom-up stimulus selection (Itti and Koch, 2001). According to these models, a salience map consists of a topographic representation of space in which neurons are not tuned to particular values of stimulus features but instead respond with increasing rates to the salience of stimuli. A winner-take-all process then selects the location in the map with the highest level of activity as the next target for gaze or attention.

Although switch-like responses are reminiscent of a winnertake-all process, these responses are not strictly winner-take-all: unlike a winner-take-all process, the response levels of switchlike neurons vary with the absolute strength of the receptive field stimulus rather than being fixed. Hence, the responses of switchlike neurons continue to encode the strength of the receptive field stimulus even when it is the "losing" stimulus. In the OT network, the winner-take-all process is represented by the activity of saccade-related neurons (Sparks, 2002). Although switch-like responses are not winner-take-all, comparing switch-like responses across the space map yields a high-resolution, binary discrimination signal of the strongest stimulus that is independent of the magnitude of the difference in stimulus strengths. The resulting representation is a step closer, computationally, to the selection of the most salient stimulus for gaze and attention (Itti and Koch, 2001). In addition, the sensory modality independence of switchlike responses suggests that the OTi-d discards the identities of stimuli and retains information only about their relative functional strengths ("salience") in the form of relative firing rates.

The OTi-d is known to project to the brainstem motor generators (Masino and Knudsen, 1992, 1993) as well as to forebrain areas involved in stimulus selection, attention, and visual processing (Shipp, 2004; Kaas and Lyon, 2007; Marín et al., 2007; Boehnke and Munoz, 2008; Reches and Gutfreund, 2009). Thus, the responses we report in the OTi-d are in a position to directly affect motor output as well as representations of relative stimulus salience in higher brain areas. Moreover, the sensory responses in the OTi-d are known to be modulated by signals from the forebrain gaze fields (Winkowski and Knudsen, 2006, 2007), allowing 
for top-down influences to modify the representation of relative stimulus salience in the OTi-d.

\section{Switch-like responses and mechanisms of competitive selection}

The competitive interactions described here for the OTi- $\mathrm{d}$ reveal functions of information processing that have not been recognized in models of sensory processing, top-down attention, or bottom-up stimulus selection (Carandini et al., 1997; Itti and Koch, 2001; Cavanaugh et al., 2002; Lee and Maunsell, 2009; Reynolds and Heeger, 2009; Olsen et al., 2010). In these models, response normalization is invoked to avoid response saturation and to adjust the sensitivity of competitive elements according to the average activation of the network. Such normalization regulates the sensitivity of "neurons" continuously across the entire range of stimulus strengths encoded by the network. This function is achieved with either divisive lateral inhibition or divisive inhibition based on pooled network activity.

The competitive rule that is expressed by gradual responses in the OTi-d is consistent with the conventional functions of response normalization: gradual responses decline systematically with the strength of competing stimuli located anywhere outside of the classical receptive field across essentially the entire range of encoded loom speeds. In contrast, the competitive rule expressed by switch-like responses, which change suddenly at a threshold (switch value) in the relative strengths of two stimuli, enhances the representation of the strongest stimulus over the representation provided by gradual responses. Such a computation serves no obvious purpose in the context of feature analysis [although models of response normalization can produce such responses using inhibitory elements with steep response functions (Carandini et al., 1997)]. However, in the context of stimulus selection, it creates a highly sensitive, explicit representation of the strongest stimulus that could be transformed in one step (comparison across pooled responses) into a reliable selection signal for controlling gaze or attention.

A decrease in response rates caused by the presence of a second stimulus is conventionally thought to be attributable to the action of a global inhibitory mechanism (Rizzolatti et al., 1974; Frost et al., 1981; Meredith and Stein, 1996; Mysore et al., 2010). Global inhibition has also been proposed to play a role in competitive selection in the OTi-d (Sereno and Ulinski, 1987; Wang, 2003; Marín et al., 2007). Moreover, a neural circuit that could mediate global inhibition in the OTi-d has been anatomically identified: GABAergic neurons in the nucleus isthmi pars magnocellularis (Imc), a satellite nucleus of the OT located in the lateral midbrain tegmentum, receive topographically organized input from the $\mathrm{OT}$ and send projections back to the OTi-d to all portions of the space map except for the portion from which they receive their input (Wang et al., 2004). However, the role of the Imc in OT processing remains to be demonstrated.

The recent discovery of the representation of relative stimulus strength in a cholinergic midbrain nucleus, called the nucleus isthmi pars parvocellularis (Ipc) (Asadollahi et al., 2010), suggests that global inhibition may only be part of the mechanism that produces response reduction in the OTi-d (Sereno and Ulinski, 1987; Wang, 2003; Marín et al., 2007). The Ipc connects with the OT in a reciprocal and precisely topographic manner. Neurons in the Ipc encode the relative strengths of competing stimuli across the entirety of space. Therefore, during stimulus competition, OTi-d neurons are influenced by strong, cholinergic modulatory input from the Ipc when the stronger stimulus is located in the receptive field and by weaker modulatory input from the
Ipc when the weaker stimulus is in the receptive field. In this manner, switch-like responses in the Ipc may accentuate switchlike responses in the OT, although the influence of the Ipc on OT responses remains unknown. Thus, inhibition from the Imc and modulatory input from the Ipc may both contribute to switchlike responses in the OTi-d.

These observations suggest that bottom-up stimulus selection in the OTi-d may involve two complementary mechanisms: a spatially precise, positive modulatory (cholinergic) mechanism that provides "push" and a global inhibitory (GABAergic) mechanism that provides "pull." The respective contributions of each of these mechanisms to stimulus selection in the OT will be the subject of additional research. However, it has been reported already that top-down signals from the forebrain gaze field also modulate the strength of sensory responses in the OTi-d in a push-pull manner (Winkowski and Knudsen, 2008). Electrical microstimulation of the forebrain gaze field focally enhances the responses of OTi-d neurons representing stimuli at the same location as that represented at the forebrain microstimulation site, while at the same time, it suppresses the responses of OTi-d neurons representing stimuli at all other locations. The coordinated action of these distinct mechanisms in top-down control of sensory responses is strikingly similar to the coordinated action of the push-pull mechanisms proposed here for bottom-up stimulus selection. These similarities support the hypothesis that bottom-up and top-down control of competitive selection share not only common principles of information processing, but they may actually share common neural circuitry in the midbrain. Moreover, the results of competitive selection in the midbrain could influence stimulus selection in the forebrain via strong, tecto-thalamic pathways (McPeek and Keller, 2004; Shipp, 2004; Marín et al., 2007; Berman and Wurtz, 2010; Lovejoy and Krauzlis, 2010).

\section{References}

Asadollahi A, Mysore SP, Knudsen EI (2010) Stimulus-driven competition in a cholinergic midbrain nucleus. Nat Neurosci 13:889-895.

Basso MA, Wurtz RH (1997) Modulation of neuronal activity by target uncertainty. Nature 389:66-69.

Berman RA, Wurtz RH (2010) Functional identification of a pulvinar path from superior colliculus to cortical area MT. J Neurosci 30:6342-6354.

Bisley JW, Goldberg ME (2003) Neuronal activity in the lateral intraparietal area and spatial attention. Science 299:81-86.

Boehnke SE, Munoz DP (2008) On the importance of the transient visual response in the superior colliculus. Curr Opin Neurobiol 18:544-551.

Carandini M, Heeger DJ, Movshon JA (1997) Linearity and normalization in simple cells of the macaque primary visual cortex. J Neurosci 17:8621-8644.

Carello CD, Krauzlis RJ (2004) Manipulating intent: evidence for a causal role of the superior colliculus in target selection. Neuron 43:575-583.

Cavanaugh JR, Bair W, Movshon JA (2002) Nature and interaction of signals from the receptive field center and surround in macaque V1 neurons. J Neurophysiol 88:2530-2546.

Cavanaugh J, Alvarez BD, Wurtz RH (2006) Enhanced performance with brain stimulation: attentional shift or visual cue? J Neurosci 26:11347-11358.

Chiu CL, Chan YK, Ong GS, Delilkan AE (2000) A comparison of the maintenance and recovery characteristic of sevoflurane-nitrous oxide against isoflurane-nitrous oxide anaesthesia. Singapore Med J 41:530-533.

Efron B, Tibshirani RJ (1994) An introduction to the bootstrap. Boca Raton, FL: Chapman and Hall/CRC.

Eger EI 2nd (2004) Characteristics of anesthetic agents used for induction and maintenance of general anesthesia. Am J Health Syst Pharm 61 [Suppl 4]:S3-S10.

Fecteau JH, Munoz DP (2006) Salience, relevance, and firing: a priority map for target selection. Trends Cogn Sci 10:382-390.

Fee MS, Mitra PP, Kleinfeld D (1996) Automatic sorting of multiple unit 
neuronal signals in the presence of anisotropic and non-Gaussian variability. J Neurosci Methods 69:175-188.

Frost BJ, Scilley PL, Wong SC (1981) Moving background patterns reveal double-opponency of directionally specific pigeon tectal neurons. Exp Brain Res 43:173-185.

Horwitz GD, Newsome WT (1999) Separate signals for target selection and movement specification in the superior colliculus. Science 284:1158-1161.

Hughes CP, Pearlman AL (1974) Single unit receptive fields and the cellular layers of the pigeon optic tectum. Brain Res 80:365-377.

Itti L, Koch C (2001) Computational modelling of visual attention. Nat Rev Neurosci 2:194-203.

Jassik-Gerschenfeld D, Guichard J (1972) Visual receptive fields of single cells in the pigeon's optic tectum. Brain Res 40:303-317.

Kaas JH, Lyon DC (2007) Pulvinar contributions to the dorsal and ventral streams of visual processing in primates. Brain Res Rev 55:285-296.

Knudsen EI (1982) Auditory and visual maps of space in the optic tectum of the owl. J Neurosci 2:1177-1194.

Knudsen EI (2011) Midbrain and forebrain systems for bottom-up control of spatial attention. In: Neuroscience of attention: attention control and selection (Mangun GR, ed). New York: Oxford UP, in press.

Lee J, Maunsell JH (2009) A normalization model of attentional modulation of single unit responses. PLoS One 4:e4651.

Li B, Wang L, Wang Y, Diao Y (1996) Orientational and directional selectivities of visual neurons in the superior colliculus of the cat. Sci China C Life Sci 39:123-132.

Lovejoy LP, Krauzlis RJ (2010) Inactivation of primate superior colliculus impairs covert selection of signals for perceptual judgments. Nat Neurosci 13:261-266.

Marín G, Salas C, Sentis E, Rojas X, Letelier JC, Mpodozis J (2007) A cholinergic gating mechanism controlled by competitive interactions in the optic tectum of the pigeon. J Neurosci 27:8112-8121.

Marrocco RT, Li RH (1977) Monkey superior colliculus: properties of single cells and their afferent inputs. J Neurophysiol 40:844-860.

Masino T, Knudsen EI (1992) Anatomical pathways from the optic tectum to the spinal cord subserving orienting movements in the barn owl. Exp Brain Res 92:194-208.

Masino T, Knudsen EI (1993) Orienting head movements resulting from electrical microstimulation of the brainstem tegmentum in the barn owl. J Neurosci 13:351-370.

McPeek RM, Keller EL (2004) Deficits in saccade target selection after inactivation of superior colliculus. Nat Neurosci 7:757-763.

Meredith MA, Stein BE (1996) Spatial determinants of multisensory integration in cat superior colliculus neurons. J Neurophysiol 75:1843-1857.

Middlebrooks JC, Knudsen EI (1984) A neural code for auditory space in the cat's superior colliculus. J Neurosci 4:2621-2634.

Mitra P, Bokil H (2008) Observed brain dynamics. New York: Oxford UP.

Müller JR, Philiastides MG, Newsome WT (2005) Microstimulation of the superior colliculus focuses attention without moving the eyes. Proc Natl Acad Sci U S A 102:524-529.
Mysore SP, Asadollahi A, Knudsen EI (2010) Global inhibition and stimulus competition in the owl optic tectum. J Neurosci 30:1727-1738.

Olsen SR, Bhandawat V, Wilson RI (2010) Divisive normalization in olfactory population codes. Neuron 66:287-299.

Reches A, Gutfreund Y (2009) Auditory and multisensory responses in the tectofugal pathway of the barn owl. J Neurosci 29:9602-9613.

Reynolds JH, Heeger DJ (2009) The normalization model of attention. Neuron 61:168-185.

Rizzolatti G, Camarda R, Grupp LA, Pisa M (1974) Inhibitory effect of remote visual stimuli on visual responses of cat superior colliculus: spatial and temporal factors. J Neurophysiol 37:1262-1275.

Schall JD, Thompson KG (1999) Neural selection and control of visually guided eye movements. Annu Rev Neurosci 22:241-259.

Sereno MI, Ulinski PS (1987) Caudal topographic nucleus isthmi and the rostral nontopographic nucleus isthmi in the turtle, Pseudemys scripta. J Comp Neurol 261:319-346.

Shipp S (2004) The brain circuitry of attention. Trends Cogn Sci 8:223-230.

Sparks DL (2002) The brainstem control of saccadic eye movements. Nat Rev Neurosci 3:952-964.

Trappenberg TP, Dorris MC, Munoz DP, Klein RM (2001) A model of saccade initiation based on the competitive integration of exogenous and endogenous signals in the superior colliculus. J Cogn Neurosci 13:256-271.

Wallace MT, Wilkinson LK, Stein BE (1996) Representation and integration of multiple sensory inputs in primate superior colliculus. J Neurophysiol 76:1246-1266.

Wang SR (2003) The nucleus isthmi and dual modulation of the receptive field of tectal neurons in non-mammals. Brain Res Brain Res Rev 41:13-25.

Wang Y, Major DE, Karten HJ (2004) Morphology and connections of nucleus isthmi pars magnocellularis in chicks (Gallus gallus). J Comp Neurol 469:275-297.

Winkowski DE, Knudsen EI (2006) Top-down gain control of the auditory space map by gaze control circuitry in the barn owl. Nature 439:336-339.

Winkowski DE, Knudsen EI (2007) Top-down control of multimodal sensitivity in the barn owl optic tectum. J Neurosci 27:13279-13291.

Winkowski DE, Knudsen EI (2008) Distinct mechanisms for top-down control of neural gain and sensitivity in the owl optic tectum. Neuron 60:698-708.

Witten IB, Knudsen PF, Knudsen EI (2010) A dominance hierarchy of auditory spatial cues in barn owls. PLoS One 5:e10396.

Wolfe JM (1994) Guided Search 2.0: a revised model of visual search. Psychonom Bull Rev 1:202-238.

Wurtz RH, Albano JE (1980) Visual-motor function of the primate superior colliculus. Annu Rev Neurosci 3:189-226.

Wurtz RH, Goldberg ME (1971) Superior colliculus cell responses related to eye movements in awake monkeys. Science 171:82-84.

Wurtz RH, Goldberg ME (1972) Activity of superior colliculus in behaving monkey. 3. Cells discharging before eye movements. J Neurophysiol 35: 575-586. 